\title{
Sports diagnosis motion capture technique
}

\author{
Mingquan Long ${ }^{1, a}$, Hongchao Cui ${ }^{1, a}$ \\ ${ }^{1}$ Physical Education Department, HuNan Vocational College of commerce, Changsha 410205, \\ Hunan, China \\ aemail: matlab_wjf@126.com
}

Keywords: Motion capture, technique and tactics, diagnosis, data processing, auxiliary training.

\begin{abstract}
The motion capture technology system definition is described in the paper, and its components are researched, the key parameters are obtained from motion technique, the quantitative analysis are made on technical movements, the method of motion capture technology is proposed in sport technical diagnosis. That motion capture step includes calibration system, to attache landmarks to the tester; to capture trajectory, and to analyze the collected data.
\end{abstract}

\section{Introduction}

For a long time, China's technological level of sports training is relatively low, teaching and training methods have been used based on subjective and experience, coaches guide the technical movements of athletes with the naked eye and experience, athletes master technology essentials only through many repetitive practice, which seriously affect further to improve our level of competitive sports.

In sports training, motion capture system can help coaches to observe and monitor the technique movement of athletes from the different perspective, and a lot of certain type technical movements and motion parameters are accessed, such as physiological and biochemical data, and its motion law is summarized to provide technical guidance for standards of scientific training. Through real-time motion capture technology, diagnosis and analysis are made for technical issues that arise intraining, and video and image as well as quantitative data are back to the coach such as the way, and the quantitative basis and quantify the training targets are provided toscientifically and accurately for coaches.

Therefore, if the motion capture technology is introducted in sports training, athletes can be helped to master action technology essentials during training, as soon as possible, to reduce the blind repetition, to greatly improve training efficiency and to reduce the possibility of injury in the body, so as to achieve the best training effect.

\section{Motion capture technology}

Motion capture technology is defined : Motion capture technology is the use of motion capture system, the athletes carry with the technical and tactical actions, or three-dimensional trajectories of moving objects are captured in real time, and it is a high-tech digital parsing [1]. Motion capture system is a high-tech equipment, the moving objects can be measured accurately in three-dimensional space movement situation, the principle is based on computer graphics, through the several video capture device arrangement in space, moving object motion position (tracker) is recorded in the form of the image, then these are processed by computer,the spatial coordinates (X, Y, Z) of the different type object (tracker) are obtained on a different unit of time[2].

Motion capture system composition : As shown in Figure 1, a typical motion capture devices are generally composed of four parts, they are the sensor, signal capture device, data transmission apparatus, data processing equipment [3]. The sensor is a tracking device, which is fixed at a specific site of the moving object, it provids the location information of moving object to the system, the tracker number depends on the detail level of motion capture. Signal capture device is a hardware device, which is responsible for collecting the relevant trajectory sensor and obtaining an 
analog signal by recognition, which is differ due to different types of systems, it is an circuit board for a mechanical system, which captures electric signal, it is a high-resolution infrared camera for the optical system. Data transmission device converts the analog signal, which is obtained by the signal capturing apparatus, into a digital signal, and the digital signal is quickly and accurately transferred to the computer system for processing. Data processing equipment consists of computer hardware and data processing software, the large amount of data are corrected, which come from the data transmission equipment, this three-dimensional modeling and animation are made. As shown in Figure 2, the current motion capture system can be divided into mechanical, voice, electromagnetic and optical from the principle[3].

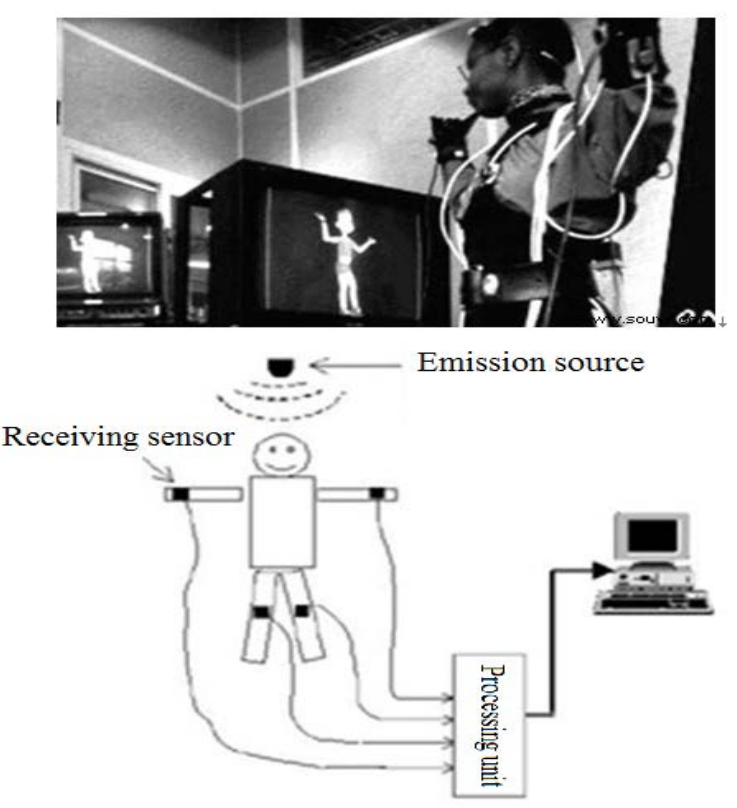

Fig.1. Motion Capture Principle

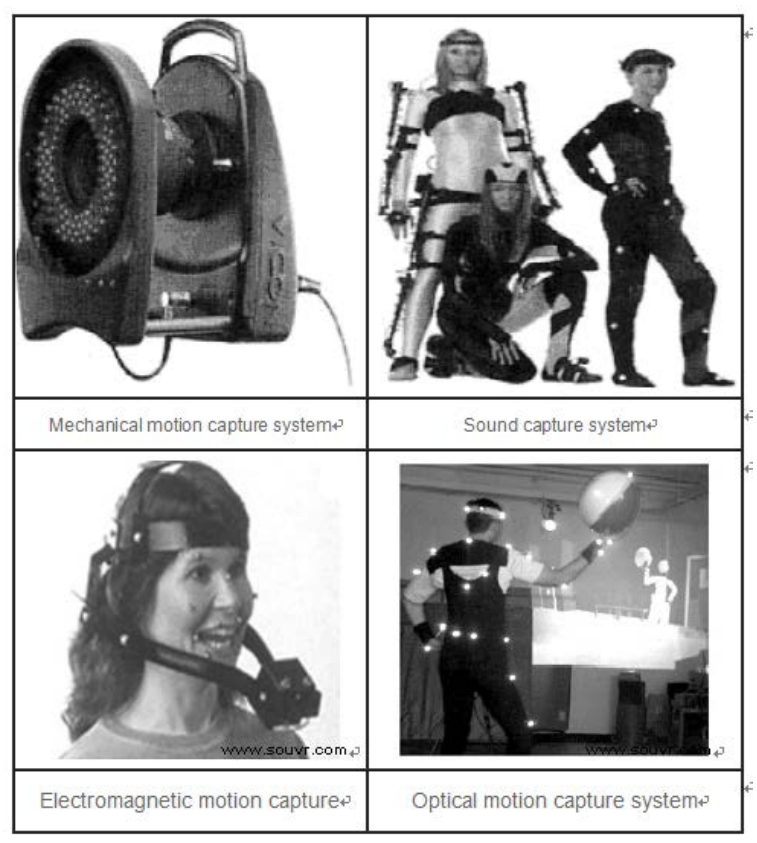

Fig.2. Various motion capture system

\section{Motion capture technology application in sport technical diagnosis}

Motion capture technology can capture and recorded the poor performance of the athlete motion, and their actions were analyzed with the elite athlete action, the quantitative training indicators 
can be provided for scientific training, and the standard and performance are raised[3].

Get motion technology key parameters : Sensor can include not only equipment to sense the movement of objects, but also measurements can be accurated on many variables, they can capture the movement position, velocity, acceleration, reaction time, distance, force, torque, power, flexion - extension, adduction - abduction , and user-defined rotation angle, its greatest advantage is the ability to capture real human data (including training equipment) of movement. Typically these sensors or transmitters are not conspicuous, it is fit for wearing, and even some information is sent to the antenna tower, and then they are propagated from these antennas. Because the formation of the movement are basically subject people or equipment movement "copy", and thus the effect is very realistic, you can make use of these data to create a dynamic data model [3]. For example, walking athletes are let to wear special clothing with mark points and special shoes, they walk on the test bench with force sensor. The image information is captured by using three CCD camera (the test system shown in Figure 3). the image data are detected, and data are processed by using Matlab programming environment, and the joint angle, the angular velocity , angular acceleration and other information are obtained (Figure 4), the data form the basis for modeling three-dimensional reconstruction of human motion.

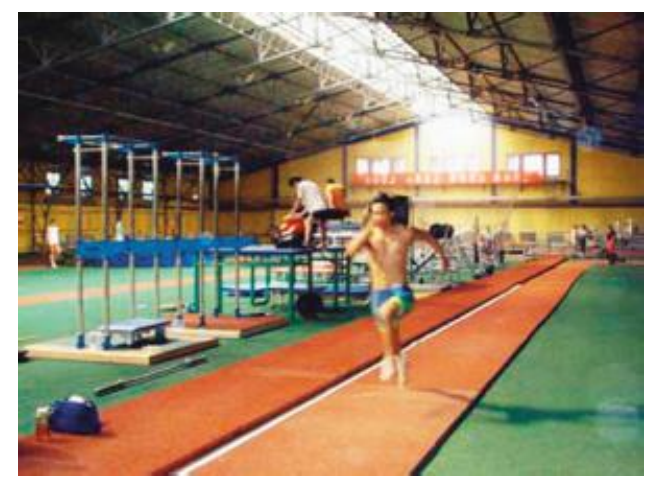

Fig.3. Walking Race Athletes Sports Technology Test System

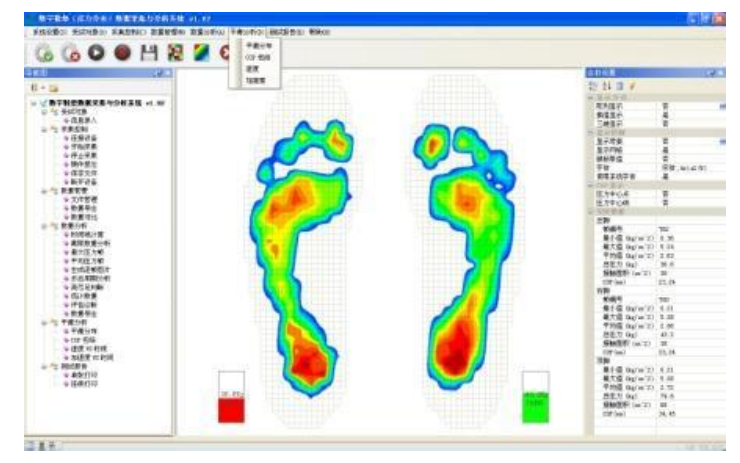

Fig.4. Walking gait cycle parameter testing and evaluation

Quantitative analysis of technical movements : With motion capture technology and computer image processing technology, the visibility of the sport can be greatly improved, and the quantitative analysis can be done beneficially to the technical action, the analysis results are graphically demonstrated, wich includs displacement, velocity and force. On this basis, the technical analysis can be done deeply between "ideal" action and athletes sport action, and the improvement guidance is given for athletes technique action[4]. As shown in Figure 5, during preparing for the 2004 Athens Olympic Games, there was a motion capture technology team in the behind of $110 \mathrm{~m}$ hurdler Liu Xiang, they bought a nearly one million worth of motion capture system from Germany,the everyday Liu Xiang training and competition were captured, as well as his main rival Alan Johnson and other technical features were captured, the captured image is digitized, these come across hurdles Liu Xiang's time for a comparative analysis with the opponent's 
data, and the findings and recommendations were feedbacked to the coaches to improve training, these lay the scientific foundation for Olympic champion Liu Xiang [5].

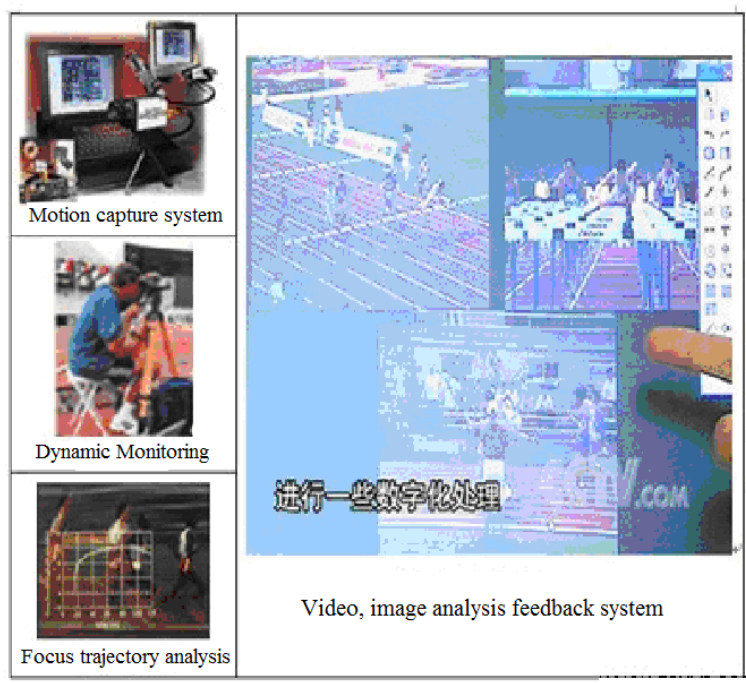

Fig.5. Liu Xiang motion capture technology

As shown in Figure 6, the assisted golf training system (GTRS-1) is a camera with image analysis type, which image is captured by, and image is processed manually by adding points, the player velocity, acceleration, angle, angular velocity are calculated at a specific joint and time. Then actions are corrected by comparison with the template data, the level of golfers are improved. By drawing an auxiliary baseline, you can visually see the player body position change during exercise, the system is mainly used for indoor courts, outdoor courts, golf stores and sports institutions and other correspondence.

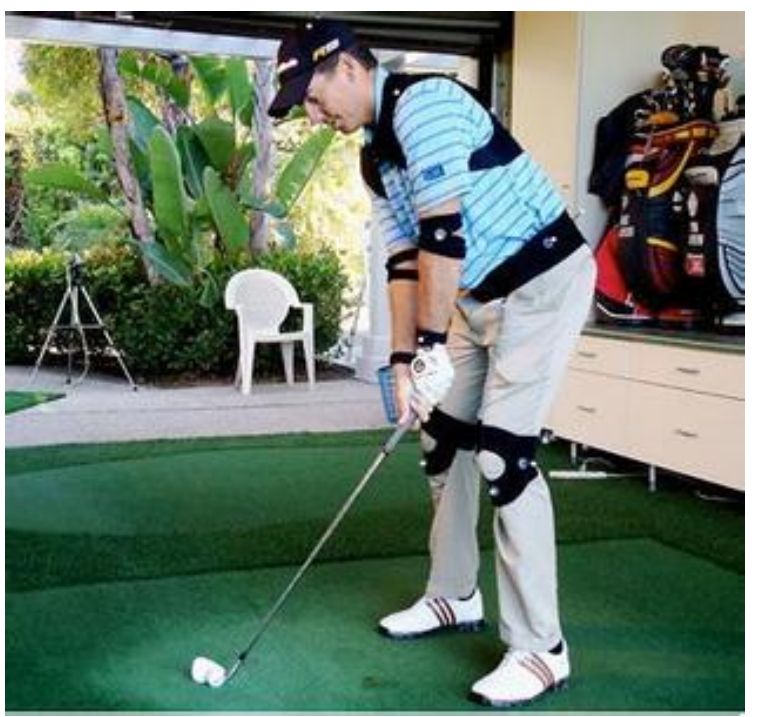

Fig.6. Golfing assisted analysis system

In weightlifting process, absolute power is important, but technical rationality is more important, through motion capture system (right in Figure 7), the force order , size, rhythm information of the athletes can be captured from the squat, grab bars, jerk to the whole process (left in Figure 7)[13-17]. 


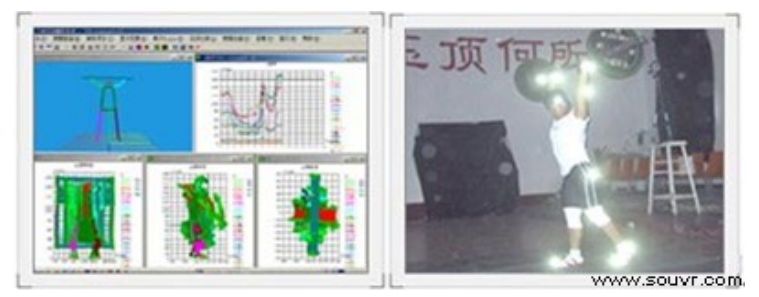

Fig. 7. Weightlifting parametric curves of capture technology(left), Weightlifting Motion Capture(right)

The system consists of data lines, sensors and recorders, computers, etc., athletes are in front of a computer screen, when their weightlifting video is playbacked, the relevant diagrams and pictures let him see his legs clearly parts of the force, the size of explosive moment, whether the bar is maintained to paralle lwith the ground during weight and etc. [6].

\section{Motion capture step and data processing}

Motion capture steps:

1) calibration system (including system setup, camera position guidelines, camera firmware versions, boardl setting, force-plate control settings, video devices)

2) marker point is posted to the tester (marker type, connection, tracking parameters, trajectories, calibration)

3) a moving trajectory is captured, and data is collected for analysis[7].

Motion capture data processing is very important in terms of technology diagnosis, how the motion capture data are effectively represent, and it is the core problem that the movement of the human body is modeled by using statistical methods, in practice, the probability model often be resulted based training, the complex human motion tracking, identification can be realized, or the realistic animation sequence are generated [8].

\section{Conclusion}

Motion capture technology can capture the action of athletes to facilitate quantitative analysis, it is combined with human physiology and physics theory, and the improvement ways are researched, physical training is made to get rid of purely relying on the experience, physical training is introduced into the scientific and digital era[10-12].

Many people believe that a variety of sports projects have reached the limit of human endurance,it has been unlikely to create a new record. However, with the help of motion capture technology, the previous Olympic record was broken from time to time, this still make you feel surprised. When you watch a moving picture on frame by frame in front of the computer, you will maybe find that the athlete swing arm is not correct, and this discovery may be just a little subtle information which coaches need to understand, posture is correct through these, athlete's speed may be improved on this a few seconds, and the athletes performance are likely to significantly enhance. It seems that this should be the plot of a science fiction movie. In fact, this is just a real-time and common motion tracking system of capturing parameters on sports field. A telemetry sensor is just install in the shoes of the player, you can observe every movement of athletes, the pressure on the muscles can be even measure on athletes each step .

At present, several challenges need to be addressed as follows: motion capture data is analyzed, high-dimensional nonlinear spatial are mapped to low-dimensional linear space, and they are effectively represented[9], and the dynamics and kinematics of the human body are analyzed, the effective modeling is made with control theory methods, the complex human movement is modeled by the statistical methods with their probability distribution. The human motion animation 
is synthesised with realistic under different constraints.

\section{References}

[1] Wang Shihua, Zhao Weifeng. IDMT Motion Capture Advanced Tutorial [M], Beijing: Beijing Hope Electronic Press, 2002:112

[2] Sun Guoyu. Motion capture technology and FilmBox basic tutorial [M], Beijing: Beijing Broadcasting Institute Press,2002:69-70

[3] FENG Lizheng, CHEN Jian. Application of Sports Catching Skill to Athletic Sports[J], JOURNAL OF HANGZHOU TEACHERS COLLEGE(MEDICAL EDITION) ,2005, 25(5),419-422

[4]Warren Robinett. Virtual Environment Workstation[OL].http://www.warrenrobinett.com,2003.

[5] Wei Tongxuan. Liu Xiang's secret weapon[EB/OL], http://www.cctv.com/sports,2004-11-12

[6] Feng Li zheng. Motion capture technology [C], the first Chinese sports simulation Institute Proceedings, Beijing, 2004:12

[7] Grigore C. Burdea, Philippe coiffet. virtual real technology[M].USA:Publisher:Wiley,John \& Sons,Incorporated,USA,2003

[8] REN C,ZHAO L,SAFONOVA A. Human motion synthesis with optimization-based graphs[J].Computer Graphics Forum (Eurographics 2010),2010,(02):545-554

[9] M(U)LLER M,R(O)DER T,CLAUSEN M. Efficient content-based retrieval of motion capture data[A].Los Angeles:ACM Press,2005.677-685.

[10] XIANG Zerui, ZHI Jinyi, XU Bochu, LI Juan. Survey on motion capture technique and its applications[J], Application Research of Computers ,2013, 30(8)

[11]LIU Zhuo, Research on Application of Somatosensary-based Motion Capture Technology in Military Sport[J], Journal of PLA Institute of Physical Education, 2013, 32(4)

[12] Zhang Junfeng, The Movement Capture Technology of Movement Training[J], JOURNAL OF JILIN INSTITUTE OF PHYSICAL EDUCATION, 2005, 21(4)

[13] Bai Xueling, Wang Hongsheng, Zhang Xian.. Three-dimensional kinematics simulation of snatch in male weight lifter and knee joint movement analysis[J],JOURNAL OF CLINICAL REHABILITATIVE TISSUE ENGINEERING RESEARCH,2009, 13(35)

[14] Carlock JM,Smith SL,Hartman MJ. The relationship between vertical jump power estimates and weightlifting ability:a field-test approach[J].Journal of Strength and Conditioning Research,2004,(03):534-539.

[15] Gourgoulis V,Aggelousis N,Mavromatis G. Three-dimensional kinematic analysis of the snatch of elite Greek weightlifters[J].Journal of Sports Sciences,2000.643-652.

[16] Hoover DL,Carlson KM,Christensen BK. Biomechanical analysis of women weightlifters during the snatch[J].Journal of Strength and Conditioning Research,2006,(03):627-633.

[17] Campos J,Poletaev P,Cuesta A. Kinematical analysis of the snatch in elite male junior weightlifters of different weight categories[J].Journal of Strength and Conditioning Research,2006,(04):843-850. 